\title{
Chronic administration of ketamine ameliorates the anxiety- and aggressive-like behavior in adolescent mice induced by neonatal maternal separation
}

\author{
Sang Yep Shin", Nam Jun Baek", Seung Ho Han*, and Sun Seek Min* \\ Department of Physiology and Biophysics, School of Medicine, Eulji University, Daejeon 34824, Korea
}

\section{ARTICLE INFO}

Received August 29, 2018

Revised October 27, 2018

Accepted November 13, 2018

\author{
*Correspondence \\ Seung Ho Han \\ E-mail: hans0424@eulji.ac.kr \\ Sun Seek Min \\ E-mail: ssmin@eulji.ac.kr
}

Key Words

Adolescence

Aggression

Anxiety

Ketamine

Maternal separation

\#These authors contributed equally to this work.

\begin{abstract}
Ketamine has long been used as an anesthetic agent. However, ketamine use is associated with numerous side effects, including flashbacks, amnesia, delirium, and aggressive or violent behavior. Ketamine has also been abused as a cocktail with ecstasy, cocaine, and methamphetamine. Several studies have investigated therapeutic applications of ketamine, demonstrating its antidepressant and anxiolytic effects in both humans and rodents. We recently reported that neonatal maternal separation causes enhanced anxiety- and aggressive-like behaviors in adolescent. In the present study, we evaluated how acute and chronic ketamine administration affected the behavioral consequences of neonatal maternal separation in adolescent mice. Litters were separated from dams for 4 hours per day for 19 days beginning after weaning. Upon reaching adolescence (post-natal day 35-49), mice were acutely (single injection) or chronically (7 daily injections) treated with a sub-anesthetic dose $(15 \mathrm{mg} / \mathrm{kg}$ ) of ketamine. At least $1 \mathrm{~h}$ after administration of ketamine, mice were subjected to open-field, elevated-plus maze, and resident-intruder tests. We found that acute ketamine treatment reduced locomotor activity. In contrast, chronic ketamine treatment decreased anxiety, as evidenced by increased time spent on open arms in the elevated-plus maze, and remarkably reduced the number and duration of attacks. In conclusion, the present study suggests that ketamine has potential for the treatment of anxiety and aggressive or violent behaviors.
\end{abstract}

\section{INTRODUCTION}

Youth violence occurs when persons aged 10-24 years, as victims, offenders, or witnesses, are involved in the intentional use of physical force or power to threaten or harm others. Youth violence is a significant public health problem with serious and lasting effects on the physical, mental, and social health of youth [1]. Many psychopathologist and neuroscientist have tried to investigate the emotional disorder such as anxiety, aggression, impulsive disorder and social deficits for common benefit.

Ketamine, a popular anesthetic agent, can be used for a variety of purposes as a painkiller, sedative, and antidepressant. A num- ber of studies have assessed the therapeutic effects of ketamine, demonstrating its antidepressant and anxiolytic effects in humans as well as in rodents [2]. However, there are lack of studies about therapeutic effects of ketamine on aggression [3].

Ketamine acts as a non-competitive antagonist of the $\mathrm{N}$ methyl-d-aspartate (NMDA) receptor. Ketamine also shows considerable interaction with voltage-sensitive $\mathrm{Ca}^{2+}$ channels, and muscarinic, monoaminergic, and opioid receptors $[4,5]$. Another property of ketamine is its potential to exert opposing effects, with different ketamine treatment regimens potentially having divergent effects on behavioral processes [6]. In particular, repeated exposure to low-dose ketamine causes persistent sup-
This is an Open Access article distributed under the terms of the Creative Commons Attribution Non-Commercial License, which permits unrestricted non-commercial use, distribution, and reproduction in any medium, provided the original work is properly cited. Copyright @ Korean J Physiol Pharmacol, pISSN 1226-4512, elSSN 2093-3827
Author contributions: S.Y.S. performed the behavioral experiments . S.Y.S. and N.J.B. wrote the manuscript. S.H.H. and S.S.M. supervised and coordinated the study. 
pression of parvalbumin (PV)- and glutamic-acid-decarboxylase 67 (GAD 67)-positive interneurons [7], and suppresses tonic glutamate input to GABAergic interneurons, resulting in disinhibition of glutamate signaling. Thus, chronic ketamine treatment by repeated injection induces excess neuronal activity. On the other hand, acute administration of ketamine, like anesthetic agents, transiently suppresses the NMDA receptor in principal neurons [8].

We recently reported that neonatal maternal separation subsequently causes enhanced anxiety- and aggressive-like behaviors in adolescent mice [9]. Furthermore, we found that these mice also exhibit reduced long-term potentiation (LTP) and release probability in the hippocampus [9] and enhanced interneuron in the dentate gyrus (our unpublished data), suggesting that altered behavior and synaptic plasticity during the adolescent period reflects changes in hippocampal interneurons. On the basis of our previous findings and the known suppressive effects of ketamine on the synthesis and action of GABAergic interneurons, we postulated that repeated ketamine injection suppresses the enhanced activity of hippocampal GABAergic interneurons and thereby affects anxiety- and aggressive-like behavior. In the present study, we sought to evaluate the effects of acute and chronic ketamine administration on anxiety- and aggressive-like behaviors induced by maternal separation in the adolescent mice.

\section{METHODS}

\section{Experimental animals}

C57BL/6 mice (Samtaco, Inc., Korea) were mated and transferred to the experimental facility at least 3 days before delivery. All mice were maintained under a 12-h light-dark cycle at a constant temperature of $21 \pm 2^{\circ} \mathrm{C}$. All mice interacted with only one researcher. Litters were randomly assigned to maternal separation (MS) and handled (HD; control) groups. All experimental procedures were performed according to Guidelines for the Care and Use of Experimental Animals, as approved by the Institutional Animal Research Ethics Committee of Eulji University (approval no. EUIACUC16-14).

\section{Maternal separation protocol}

The maternal separation protocol is described in detail in previous papers [9-11]. Briefly, MS-group litters were separated from dams for $4 \mathrm{~h}$ per day from post-natal day 2 (PND2) to PND20 [1012]. Litters were returned to their home cage after the 4-h separation period. HD group animals were similarly handled, but were not separated. All litters were weaned at PND22 and then separated according to gender and treatment. Subsequent procedures were carried out using males only [13]. After weaning, group sizes were standardized (3-5 males/cage), and mice were maintained under these conditions until tested. Tests were performed during adolescence (PND35-49) using 7-10 mice per condition.

\section{Open-field test}

An open-field test ( $50 \times 50 \mathrm{~cm}$ arena) was used to measure spontaneous locomotor behavior. Mice were placed in the center of the field, and their activity was recorded for 5 min using an Ethovision video-tracking system (EVTS; Noldus, Leesburg, VA, USA).

\section{Elevated plus-maze test}

Each mouse was placed in the center of the maze (open arm: $65 \times 5 \mathrm{~cm}$; closed arm: $65 \times 5 \mathrm{~cm}$; height: $15 \mathrm{~cm}$ ) and then allowed to search the open and closed arms of the maze for $10 \mathrm{~min}$. The amount of time spent in closed and open arms, recorded using the EVTS, was divided by the total time spent on the apparatus (600 s). The number of entrances into open arms or closed arms was divided by the total number of entrances into either arm.

\section{Resident-intruder test}

Adolescent male mice were isolated in their cages for 2 weeks before experiments. On the day of the experiment, an agematched male mouse of a different species (Balb/c male) was placed in the cage, and aggressive-like behaviors were recorded for 10 min using the EVTS. The total number of attacks, duration of attacks, and latency to the first attack were analyzed.

\section{Drugs and treatment}

After the neonatal maternal separation procedure (PND220), adolescent mice (PND35-49) were administered a single intraperitoneal injection of a sub-anesthetic dose $(15 \mathrm{mg} / \mathrm{kg})$ of ketamine (Sigma Chemical Co., St. Louis, MO, USA) (acute treatment) or a daily injection of the same dose for 1 week (chronic treatment). Mice were injected with a total volume of $1 \mathrm{ml} / \mathrm{kg}$. The dose of ketamine employed in this work was chosen based on previous studies $[14,15]$. The present study employed 138 mice assigned to six groups: 1) HD+saline; 2) HD+acute ketamine; 3) $\mathrm{HD}+$ chronic ketamine; 4) MS+saline; 5) MS+acute ketamine; and 6) MS+chronic ketamine. At least $1 \mathrm{~h}$ after administration of ketamine, mice were subjected to open-field, elevated-plus maze, and resident-intruder tests. Open-field and elevated-plus maze tests were performed sequentially at each mice (PND42). Resident-intruder test (RIT) was performed at the other mice (PND49) (Fig. 1).

\section{Statistics}

Data were analyzed using SPSS version 10.0 software (SPSS Inc., Chicago, IL, USA). All values are given as means \pm SEM. Sta- 

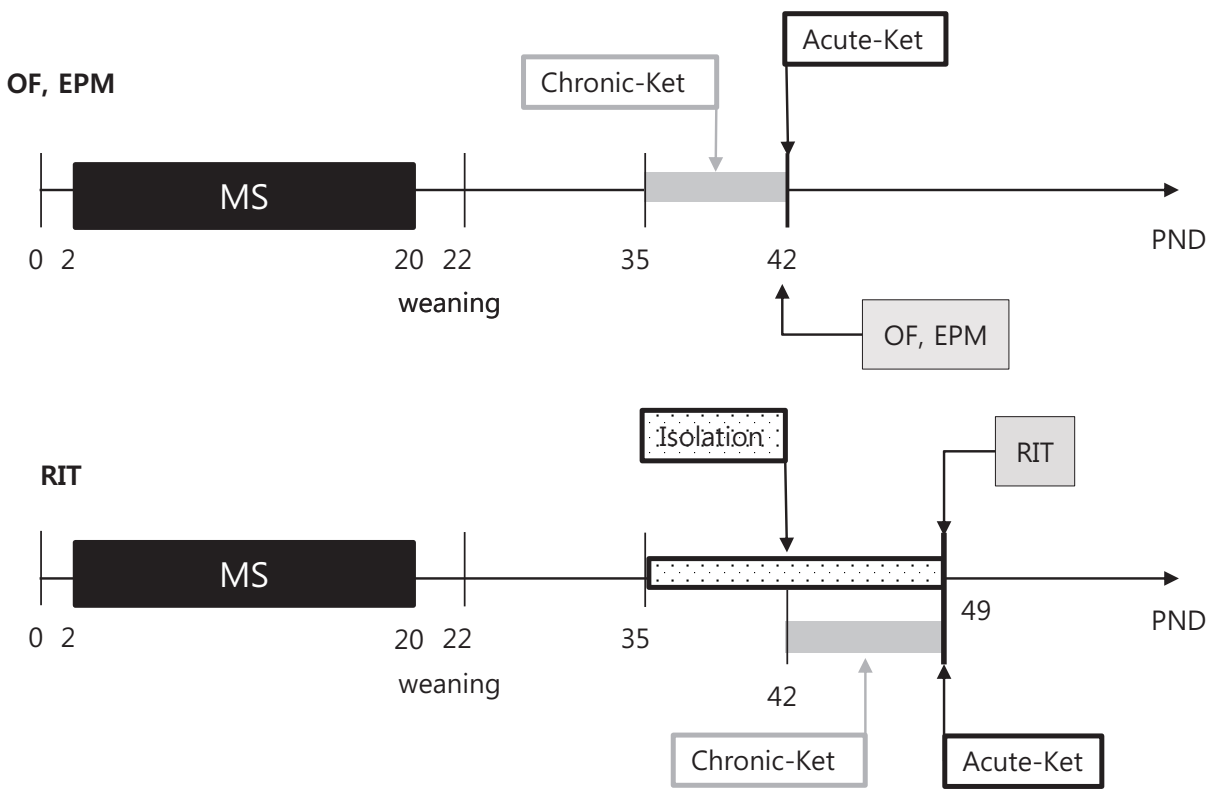

Fig. 1. Experimental timeline for behavioral tests. Litters were randomly assigned to maternal separation (MS) and handled (HD; control) groups. After the neonatal maternal separation procedure (PND2-20), all litters were weaned at PND22 and then separated according to gender and treatment. Adolescent mice (PND35-49) were administered a single intraperitoneal injection of a sub-anesthetic dose of ketamine (acute treatment) or a daily injection of the same dose for 1 week (chronic treatment). Open-field (OF) and elevated-plus maze (EPM) tests were performed sequentially at each mice (PND42). Resident-intruder test (RIT) was performed at the other mice (PND49). Before RIT, adolescent male mice were isolated in their cages for 2 weeks (PND35-49). At least $1 \mathrm{~h}$ after administration of ketamine, mice were subjected to OF, EPM test and RIT.

tistical significance was assessed using one-way analysis of variance (ANOVA) followed by Tukey's post hoc test. p-values $<0.05$ were considered statistically significant.

\section{RESULTS}

Locomotor behavior was assessed using the open-field test. As shown Fig. 2, the velocity and distance moved by mice in MS groups treated with saline (MS+Sal) or chronically treated with ketamine (MS+Chronic Ket) were not significantly different from those of mice in the HD group treated with saline. By contrast, the total distance moved following acute treatment with ketamine was significantly reduced compared with that following saline treatment in HD mice (HD+Acute Ket, $1648.7 \pm 110.4 \mathrm{~cm}$ vs. $\mathrm{HD}+\mathrm{Sal}, 2936.3 \pm 110.1 \mathrm{~cm} ; \mathrm{p}<0.001)$ and MS mice (MS+Acute Ket, $1726.0 \pm 117.0 \mathrm{~cm}$ vs. MS+Sal, $2810.0 \pm 144.3 \mathrm{~cm}, \mathrm{p}<0.001$; Fig. 2A). Compared with saline treatment, acute treatment with ketamine similarly reduced velocity in $\mathrm{HD}$ mice (HD+Acute Ket, $2.75 \pm 0.18 \mathrm{~cm} / \mathrm{s}$ vs. HD+Sal, $4.89 \pm 0.18 \mathrm{~cm} / \mathrm{s} ; \mathrm{p}<0.001)$ and $\mathrm{MS}$ mice (MS+Acute Ket, $2.88 \pm 0.20 \mathrm{~cm} / \mathrm{s}$ vs. MS+Sal, $4.69 \pm 0.24 \mathrm{~cm} / \mathrm{s}$; p $<0.001$; Fig. 2B).

In the elevated plus-maze test, used to assess anxiety-like behaviors, the percentage of time spent in open arms for mice in the MS+Sal group $(2.6 \% \pm 0.7 \%)$ was less than that for mice in the $\mathrm{HD}+$ Sal group $(9.7 \% \pm 1.2 \%$; $<<0.05$; Fig. 3 ). The percentage of closed arm entries was also higher for mice in the MS+Sal group

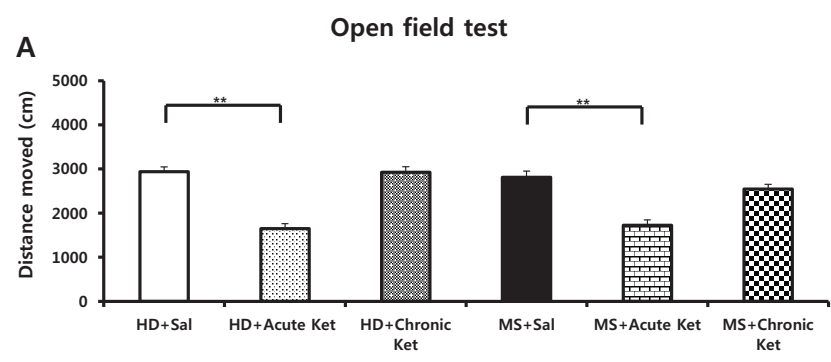

B

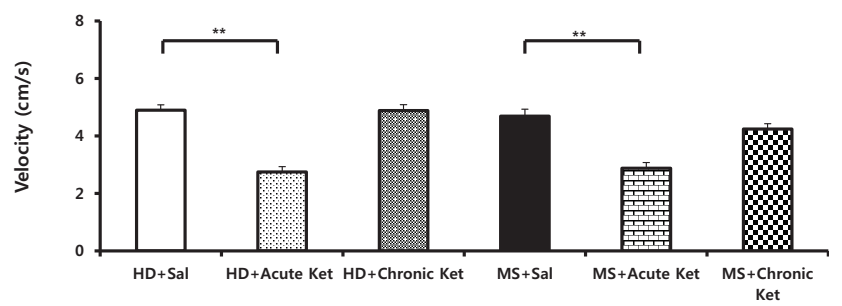

Fig. 2. Effects of maternal separation on open-field test performance in mice acutely or repeatedly treated with ketamine (15 $\mathbf{m g} / \mathbf{k g}$ ). (A) Distanced moved and (B) velocity were recorded in an acrylic cage $\left(30 \mathrm{~cm}^{2}\right)$. Results are expressed as means \pm SEM (HD group, $\mathrm{n}=23$; MS group, $\mathrm{n}=24 ;{ }^{* *} \mathrm{p}<0.001$ vs. HD+Sal, ${ }^{* *} \mathrm{p}<0.001$ vs. MS+Sal; one-way ANOVA with Tukey's post hoc test). 
$(75.7 \% \pm 3.4 \%)$ than for mice in the HD+Sal group $(87.2 \% \pm 2.5 \%$; $\mathrm{p}<0.05$; Fig. 3). The reduction in time spent in open arms was further exacerbated by acute treatment with ketamine. Specifically, the percentage of time spent in open arms was reduced to
$3.2 \% \pm 1.15 \%$ for mice in the HD+Acute Ket group (vs. $9.7 \pm 1.2 \%$ in the HD+Sal group; $\mathrm{p}<0.001$; Fig. 3), and was dramatically decreased to $0.4 \% \pm 0.2 \%$ in the MS+Acute Ket group (vs. $2.6 \% \pm 0.7 \%$ in the MS+Sal group; $\mathrm{p}<0.01$, Fig. 3). In contrast, chronic treat-

\section{Elevated Plus maze}

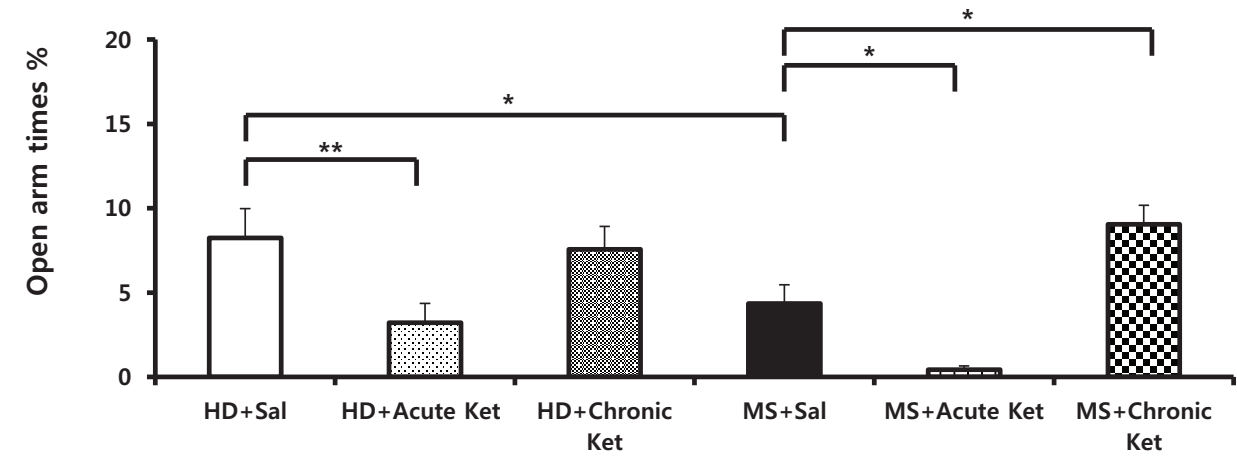

Fig. 3. Effects of maternal separation on elevated plus-maze performance in mice acutely or repeatedly treated with ketamine (15 mg/kg). Anxiety-like behavior was reflected in the percentage of time spent in open arms of the elevated plus maze. Results are expressed as means \pm SEM (HD group, $\mathrm{n}=22$; MS group, $n=26 ;{ }^{*} p<0.05,{ }^{* *} p<0.001$ vs. $\mathrm{HD}+\mathrm{Sal} ;{ }^{*} \mathrm{p}<0.05,{ }^{* *} \mathrm{p}<0.001$ vs. MS+Sal; one-way ANOVA followed by Tukey's post hoc test).

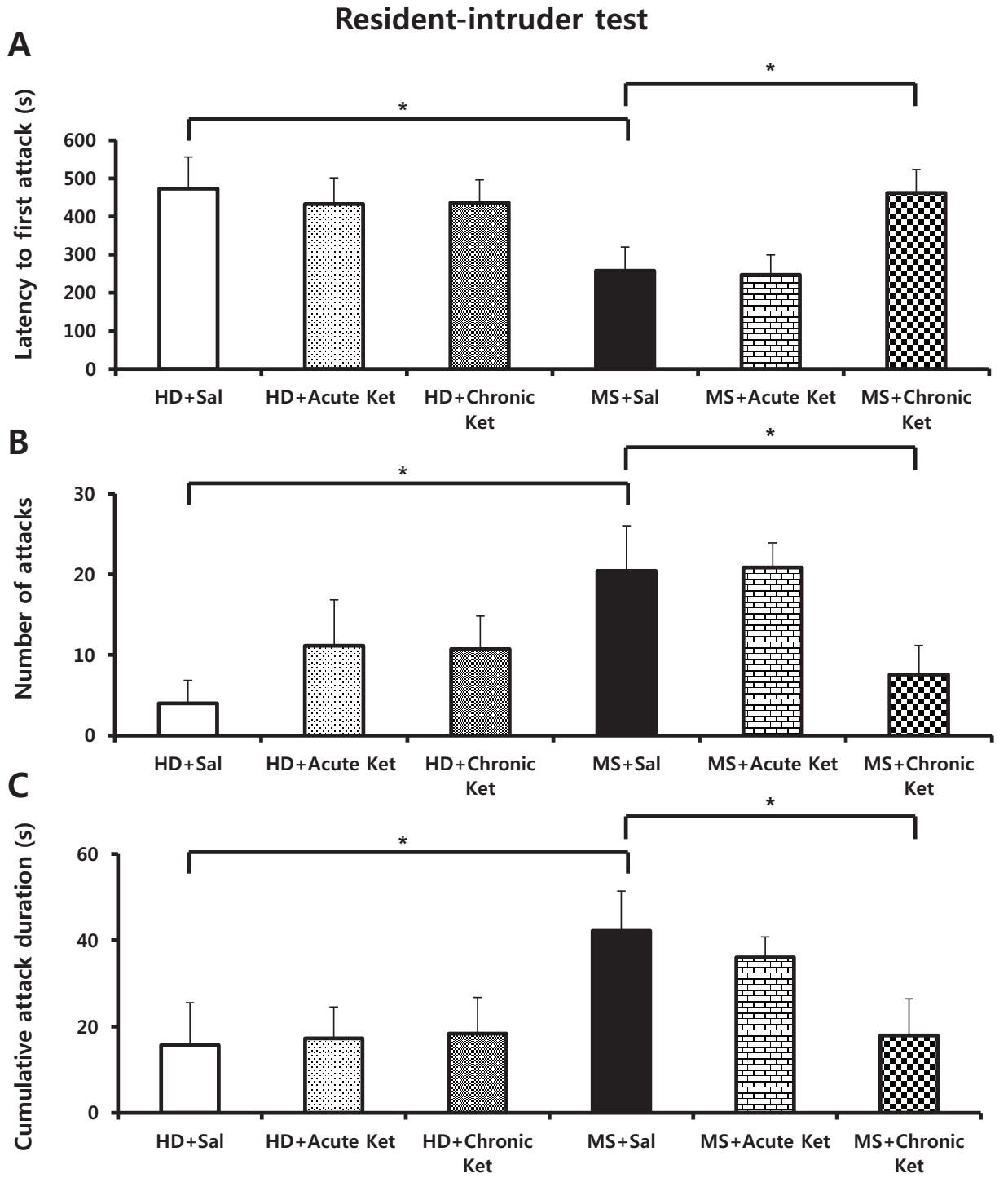

Fig. 4. Effects of maternal separation on performance in the resident-intruder test in mice acutely or repeatedly treated with ketamine $(15 \mathrm{mg} /$ $\mathbf{k g})$. Aggressive-like behavior was measured as the latency to first attack (A), number of attacks (B), and cumulative attack duration (C). Results are expressed as means \pm SEM (HD group, $\mathrm{n}=21$; MS group, $\mathrm{n}=22 ;{ }^{*} \mathrm{p}<0.05$ vs. HD+Sal, ${ }^{*} \mathrm{p}<0.05$ vs. MS+Sal; one-way ANOVA followed by Tukey's post hoc test). 
ment with ketamine exerted a reversing effect such that mice in the MS+Chronic Ket group spent a greater percentage of time in open arms $(9.05 \% \pm 2.43 \%)$ compared with mice in the MS+Sal group (2.58 $\pm 0.71 \%$; $\mathrm{p}<0.05$; Fig. 3 ).

Finally, we measured aggressive behavior using the residentintruder test. The latency to the first attack of an intruder was shorter in the MS+Sal group (214.3 \pm 52.3 seconds) than in the HD+Sal group ( $541.4 \pm 58.6$ seconds; $\mathrm{p}<0.05$; Fig. $4 \mathrm{~A})$. The number of attacks was also more frequent in the MS+Sal group (26.20 \pm 5.82$)$ than in the $\mathrm{HD}+$ Sal group $(4.00 \pm 2.84 ; \mathrm{p}<0.05$, Fig. $4 \mathrm{~B}$ ). The cumulative attack duration was also longer in the MS+Sal group (48.24 \pm 8.21 seconds) than in the HD+Sal group (6.39 \pm 4.07 seconds; $\mathrm{p}<0.05$; Fig. 4C). However, chronic treatment of MS mice with ketamine rescued the reduced latency to attack (462.1 \pm 61.5 vs. $214.3 \pm 52.3$ seconds for MS+Chronic Ket and MS+Sal, respectively; $\mathrm{p}<0.05$, Fig. $4 \mathrm{~A}$ ), increased number of attacks (5.50 \pm 3.50 vs. $26.20 \pm 5.82$ for MS+Chronic Ket and MS+Sal, respectively; $<<0.05$; Fig. 4B) and duration of attack (12.99 \pm 8.23 vs. 48.24 \pm 8.21 seconds for MS+Chronic Ket and MS+Sal, respectively; $\mathrm{p}<0.05$; Fig. 4C). Acute treatment with ketamine did not influence aggressive-like behavior in either the HD or MS group.

\section{DISCUSSION}

In the present study, we demonstrated that (1) MS mice exhibit increased anxiety; (2) MS mice display an increase in aggressivelike behavior; (3) chronic, but not acute, treatment with ketamine reverses the anxiety and aggressive-like behavior induced by maternal separation.

Maternal separation translates to a deficiency of proper maternal care and guidance, and can induce profound neurochemical, cognitive, and emotional dysfunction [16]. Maternal separation in childhood accounts for an increased rate of subsequent depression and anxiety [17]. Our study showed that maternal separation of mice as neonates induces aggression and anxiety during adolescence, consistent with the results of our previous study [9]. When neonates encounter a stressful environment, such as protracted separation from the dam, it causes a deterioration in the adult's response to stressors. These early-life experiences affect Hypothalamic-Pituitary-Adrenal (HPA) axis functions, such as negative feedback derived from stimulation of hippocampal glucocorticoid receptors [18].

The hippocampus, which is critical for the HPA axis, appears to be particularly vulnerable. Maternal separation causes structural plasticity in two ways: it drives atrophy of dendrites of pyramidal cells in the CA3 region, and restrains neurogenesis of dentate gyrus granule neurons. Consistent with this, the magnitude of LTP in mice in the MS group is decreased at mossy fiber (MF)-CA3 synapses [9]. We suggest that maternal separation modifies the structural plasticity of the hippocampal CA3 region and affects the HPA axis-effects that collectively worsen the response to a stressor and manifest as aggression and anxiety.

Ketamine has long been used as an anesthetic agent. However, ketamine use is associated with numerous side effects, including flashbacks, amnesia, delirium, and aggressive or violent behavior; it is also abused as part of a cocktail with ecstasy, cocaine, and methamphetamine. In terms of therapeutic effects, several studies have demonstrated that ketamine has antidepressant and anxiolytic effects in humans as well as in rodents [2].

The structure and effects of ketamine, such as the high incidence of post-anesthetic emergence phenomena and abuse potential, resembles those of phencyclidine (PCP) [19]. It is therefore reasonable to assume that ketamine could induce other psychopharmacological effects similar to those produced by PCP, including aggressive behavior [20]. However, there is considerable debate regarding the impact of ketamine on aggression. Previous studies showed that low doses of ketamine (3 and $5 \mathrm{mg}$ / $\mathrm{kg}$ ) enhanced aggressive behavior in rats deprived of rapid eye movement (REM) sleep and in food-deprived animals pretreated with apomorphine. However, it appeared that $3 \mathrm{mg} / \mathrm{kg}$ ketamine significantly inhibited mouse-killing behavior in rats previously demonstrated to exhibit muricidal behavior [20]. In each case, these results were obtained after acute administration of ketamine, prompting questions about whether chronic ketamine administration affects aggressive behavior.

Our findings showed that acute administration of a subanesthetic dose of ketamine $(15 \mathrm{mg} / \mathrm{kg})$ to mice in the MS group decreased locomotor activity (both distance moved and velocity), enhanced anxiety-like behavior in the elevated plus-maze test (based on the low open arm/closed arm ratio), but had no significant effect on aggression-like behavior in a resident-intruder test compared with mice in the MS+Sal group. In contrast, we found that chronic administration (7 days) of the same dose of ketamine had no significant effect on locomotor activity, but decreased both anxiety-like behavior (elevated plus-maze test) and aggression-like behavior (resident-intruder test) compared with mice in the MS+Sal group.

NMDA receptor blockade, for example with ketamine, effectively prevents stress-induced dendritic atrophy in the CA3 region [21]. Therefore, it can be inferred that ketamine restores the synaptic plasticity of the hippocampal CA3 region by promoting neurogenesis [22]. Restoration of synaptic plasticity resets the HPA axis to normal, resulting in a resistant response to a stressor. Resistant responses to a stressor, in turn, decrease the levels of aggression and anxiety [23]. However, acute administration of ketamine in the present study did not decrease aggression or anxiety in mice in the MS group. Restoration of LTP and dendritic atrophy requires repetitive synaptic stimulation [24,25]. Taken together with the fact that repeated, but not acute, ketamine administration increases adult hippocampal neurogenesis, these observations suggest that repeated ketamine administration would result in greater behavioral consequences than acute treatment [22]. 
Several studies have specifically investigated the antidepressant and anxiolytic effects of acute administration of ketamine. However, there are lack of studies about anti-aggressive effect of ketamine to rodent. Recently, one study reported lower sub-anesthetic concentrations $(2 \mathrm{mg} / \mathrm{L})$ increase aggression towards mirror, higher ketamine concentrations $(20 \mathrm{mg} / \mathrm{L}$ and $40 \mathrm{mg} / \mathrm{L}$ ) elicit stereotypic behaviors and reduce aggression for adult zebrafish [3]. Another study showed acute ketamine (7.5 and $10.0 \mathrm{mg} / \mathrm{kg}$ ) suppressed aggressive performance in both control group and in historically alcohol-treated group of adult mice (PND 8 weeks) [26].

In our study, which focused on chronic administration, we found that repeated ketamine injections increased the time spent on open arms and induced a remarkable reduction in the number and duration of attacks. Therefore, these findings suggest that ketamine has therapeutic potential for the treatment of anxiety and aggressive or violent behavior. Given the fact that maternal separation causes borderline personality [27] and antisocial behavior [28], and these disorders require long term treatment [29], it is important to study therapeutic effect of chronic ketamine in the maternal separation model. Since our data are limited to behavioral study, more extensive studies are necessary to further elucidate the mechanisms of anti-aggressive effects of ketamine by using molecular and electrophysiological methods.

\section{ACKNOWLEDGEMENTS}

This research was supported by the Bio \& Medical Technology Development Program of the National Research Foundtion (NRF) \& funded by the Korean government (MSIP\&MOHW) (No. 2016M3A9B6904244) and NRF(2015R1D1A1A01061326).

\section{CONFLICTS OF INTEREST}

The authors declare no conflicts of interest.

\section{REFERENCES}

1. David-Ferdon C, Simon TR, Spivak H, Gorman-Smith D, Savannah SB, Listenbee RL, Iskander J. Centers for Disease C, Prevention. CDC grand rounds: preventing youth violence. MMWR Morb Mortal Wkly Rep. 2015;64:171-174.

2. Maeng S, Zarate CA Jr. The role of glutamate in mood disorders: results from the ketamine in major depression study and the presumed cellular mechanism underlying its antidepressant effects. Curr Psychiatry Rep. 2007;9:467-474.

3. Michelotti P, Quadros VA, Pereira ME, Rosemberg DB. Ketamine modulates aggressive behavior in adult zebrafish. Neurosci Lett. 2018;684:164-168.

4. Hirota K, Lambert DG. Ketamine: its mechanism(s) of action and unusual clinical uses. Br J Anaesth. 1996;77:441-444.

5. Bolshakov KV, Gmiro VE, Tikhonov DB, Magazanik LG. Determinants of trapping block of N-methyl-d-aspartate receptor channels. J Neurochem. 2003;87:56-65.

6. Brown JA, Ramikie TS, Schmidt MJ, Báldi R, Garbett K, Everheart MG, Warren LE, Gellért L, Horváth S, Patel S, Mirnics K. Inhibition of parvalbumin-expressing interneurons results in complex behavioral changes. Mol Psychiatry. 2015;20:1499-1507.

7. Behrens MM, Ali SS, Dao DN, Lucero J, Shekhtman G, Quick KL, Dugan LL. Ketamine-induced loss of phenotype of fast-spiking interneurons is mediated by NADPH-oxidase. Science. 2007;318:16451647.

8. MacDonald JF, Miljkovic Z, Pennefather P. Use-dependent block of excitatory amino acid currents in cultured neurons by ketamine. $J$ Neurophysiol. 1987;58:251-266.

9. Shin SY, Han SH, Woo RS, Jang SH, Min SS. Adolescent mice show anxiety- and aggressive-like behavior and the reduction of longterm potentiation in mossy fiber-CA3 synapses after neonatal maternal separation. Neuroscience. 2016;316:221-231.

10. Andersen SL, Lyss PJ, Dumont NL, Teicher MH. Enduring neurochemical effects of early maternal separation on limbic structures. Ann N Y Acad Sci. 1999;877:756-759.

11. Liu D, Caldji C, Sharma S, Plotsky PM, Meaney MJ. Influence of neonatal rearing conditions on stress-induced adrenocorticotropin responses and norepinepherine release in the hypothalamic paraventricular nucleus. J Neuroendocrinol. 2000;12:5-12.

12. Andersen SL, Teicher MH. Delayed effects of early stress on hippocampal development. Neuropsychopharmacology. 2004;29:19881993.

13. Bath KG, Chuang J, Spencer-Segal JL, Amso D, Altemus M, McEwen BS, Lee FS. Variant brain-derived neurotrophic factor (Valine66Methionine) polymorphism contributes to developmental and estrous stage-specific expression of anxiety-like behavior in female mice. Biol Psychiatry. 2012;72:499-504.

14. Chen YW, Sherpa AD, Aoki C. Single injection of ketamine during mid-adolescence promotes long-lasting resilience to activity-based anorexia of female mice by increasing food intake and attenuating hyperactivity as well as anxiety-like behavior. Int J Eat Disord. 2018;51:1020-1025.

15. Nagy LR, Featherstone RE, Hahn CG, Siegel SJ. Delayed emergence of behavioral and electrophysiological effects following juvenile ketamine exposure in mice. Transl Psychiatry. 2015;5:e635.

16. Li M, Xue X, Shao S, Shao F, Wang W. Cognitive, emotional and neurochemical effects of repeated maternal separation in adolescent rats. Brain Res. 2013;1518:82-90.

17. Harris T, Brown GW, Bifulco A. Loss of parent in childhood and adult psychiatric disorder: the role of lack of adequate parental care. Psychol Med. 1986;16:641-659.

18. Anisman H, Zaharia MD, Meaney MJ, Merali Z. Do early-life events permanently alter behavioral and hormonal responses to stressors? Int J Dev Neurosci. 1998;16:149-164.

19. White PF, Way WL, Trevor AJ. Ketamine--its pharmacology and therapeutic uses. Anesthesiology. 1982;56:119-136.

20. Takahashi RN, Morato GS, Monteiro-de-Lima TC. Effects of ketamine on experimental animal models of aggression. Braz J Med Biol Res. 1984;17:171-178.

21. McEwen BS, Albeck D, Cameron H, Chao HM, Gould E, Hastings 
N, Kuroda Y, Luine V, Magariños AM, McKittrick CR, Orchinik M, Pavlides C, Vaher P, Watanabe Y, Weiland N. Stress and the brain: a paradoxical role for adrenal steroids. Vitam Horm. 1995;51:371-402.

22. Clarke M, Razmjou S, Prowse N, Dwyer Z, Litteljohn D, Pentz R, Anisman H, Hayley S. Ketamine modulates hippocampal neurogenesis and pro-inflammatory cytokines but not stressor induced neurochemical changes. Neuropharmacology. 2017;112:210-220.

23. Neumann ID, Veenema AH, Beiderbeck DI. Aggression and anxiety: social context and neurobiological links. Front Behav Neurosci. 2010;4:12. doi: 10.3389/fnbeh.2010.00012.

24. Magariños AM, Verdugo JM, McEwen BS. Chronic stress alters synaptic terminal structure in hippocampus. Proc Natl Acad Sci U S A. 1997;94:14002-14008.

25. Baudry M, Oliver M, Creager R, Wieraszko A, Lynch G. Increase in glutamate receptors following repetitive electrical stimulation in hippocampal slices. Life Sci. 1980;27:325-330.

26. Covington HE 3rd, Newman EL, Tran S, Walton L, Hayek W, Leonard MZ, DeBold JF, Miczek KA. The urge to fight: persistent escalation by alcohol and role of NMDA receptors in mice. Front Behav Neurosci. 2018;12:206. doi: 10.3389/fnbeh.2018.00206

27. Crawford TN, Cohen PR, Chen H, Anglin DM, Ehrensaft M. Early maternal separation and the trajectory of borderline personality disorder symptoms. Dev Psychopathol. 2009;21:1013-1030.

28. Haller J, Harold G, Sandi C, Neumann ID. Effects of adverse earlylife events on aggression and anti-social behaviours in animals and humans. J Neuroendocrinol. 2014;26:724-738.

29. Kendall T, Pilling S, Tyrer P, Duggan C, Burbeck R, Meader N, Taylor C; Guideline Development Groups. Borderline and antisocial personality disorders: summary of NICE guidance. BMJ. 2009;338:b93. doi: 10.1136/bmj.693. 